Niniejsza publikacja jest dostępna na licencji Creative Commons. Uznanie autorstwa-Użycie niekomercyjne-Bez utworów zależnych 3.0 Polska. Pewne prawa zastrzeżone na rzecz autora. Zezwala się na wykorzystanie publikacji zgodnie z licencja - pod warunkiem zachowania niniejszej informacji licencyjnej oraz wskazania autora jako właściciela praw do tekstu. Treść licencji jest dostęna na stronie: http://creativecommons.org/licenses/by-nc-nd/3.0/pl/

Lingwistyka Stosowana 19: 4/2016, 137-147

\author{
Emil Daniel LESNER
}

Uniwersytet Szczeciński

\title{
Zum Übersetzungsproblem melischer Texte am Beispiel des Lieds „Wilcza zamieć" und seiner deutschen Übertragung
}

\begin{abstract}
:
The problem of translating melical texts on example of song ,Wilcza zamiećc6 and its German rendition

The author discusses the basic problems of translation of melical texts. He shows also on example of a song "Wilcza zamieć" and its German rendition the translation techniques and strategies, which can be implemented in the translation process.
\end{abstract}

\section{Einleitung}

Die Dichtung wird laut Meinungen vieler Theoretiker (man kann hier u.a. die Beiträge von Walter Benjamin ( W. Benjamin 1923) oder Roman Jakobson (R. Jakobson 1959) nennen) als ein schwieriges Übersetzungsproblem angesehen. Der besondere Platz soll dabei ihren melischen Eigenschaften gewidmet werden. Die Bestimmung der Texte für das Singen verursacht, dass der Übersetzer nicht alle zur Verfügung stehenden Übersetzungstechniken anwenden kann. Zu anderen Schwierigkeiten, die bei der Wiedergabe solcher Texte auftreten, gehören die Reimstruktur des Ausgangstextes und die Intertextualität ${ }^{1}$. Das Ziel des vorliegenden Beitrags ist die qualitative Bewertung der angewandten Übersetzungstechniken bei der Übertragung des Liedes "Wilcza zamieć" ins Deutsche. Das Lied entstammt dem polnischen Computerspiel "Wiedźmin 3: Dziki Gon". Untersucht und beschrieben werden sowohl formale und semantische Aspekte des Ausgangstextes (darunter auch implizite Inhalte) als auch die Methoden ihrer Wiedergabe in der deutschen Sprache.

\section{Das Lied als ein audiomedialer, formbetonter Text}

Das Lied, der singende, oft mit Musik begleitete Vortrag, ist die älteste Form der Lyrik, ja aller Dichtung überhaupt: Damit der dichterische Text sich dem Gedächtnis der Singenden wie der Zuhörenden besser einprägte als die Prosa, wurde er in Versen verfasst und erhielt eine musiknahe Rhytmik und Metrik (D. Burdorf 1997: 22f.)

\footnotetext{
${ }^{1}$ Ein neues Intertextualitätmodell wird im Jahre 2016 von Sulikowski vorgeschlagen (vgl. P. R. Sulikowski 2016).
} 
Das oben angeführte Zitat stellt den Begriff des Liedes mit dem Begriff der Dichtung zusammen. Dabei entsteht die Frage, ob Liedtexte auf dieselbe Weise wie poetische Texte gestaltet werden, was von besonderer Bedeutung für die Wahl einer passenden Übersetzungstechnik und für den ganzen Übersetzungsprozess ist. Vor der Übersetzungsanalyse wäre dementsprechend erforderlich einige Unterschiede zwischen einem Liedtext und einem poetischen Text zu erläutern ${ }^{2}$. Ähnlich wie andere Texte, darunter künstlerische Texte wie Gedichte oder Prosa (vgl. R. Jakobson 1960: 88), werden die Lieder als linguistische Mitteilungen angesehen. Sie werden durch einen Sender einem bestimmten Empfänger mitgeteilt, nehmen Bezug auf einen entsprechenden Kontext und werden in einem entsprechenden Kode (d.h. in einer bestimmten, für die beiden Kommunikationspartner verständlichen Sprache) ausgedrückt.

Der Unterschied zwischen einem Lied und einem poetischen Text besteht im Rezeptionskanal. Im Falle eines Liedes haben wir mit dem akustischen Kanal und im Falle der traditionellen Dichtung mit dem visuellen Kanal zu tun. Die dominante Sprachfunktion der beiden ist die sog. poetische Funktion der Sprache (vgl. ebenda: 92). Jakobson stellt fest, dass sie „das Prinzip der Äquivalenz von der Achse der Selektion auf die Achse der Kombination" projiziere (vgl. ebenda: 94). Damit wird nämlich die Wiederholbarkeit bestimmter Spracheinheiten gemeint und die Möglichkeit sie mit anderen wiederholbaren Eigenschaften zu verbinden. In Bezug auf Lieder werden Strophen, Refrain, Akzentsetzung in einer bestimmten Zeile des Textes (sie hängt allerdings noch von der musikalischen Gestaltung des Textes ab, wo die Silbenbetonung in Sätzen an die komponierte Musik angepasst werden sollte) und die reimische Struktur wiederholbar. Man kann vermuten, dass die o. g. Akzentsetzung aus pragmatischer Sicht zu den wichtigsten, textstiftenden Merkmalen eines Musikstückes gehört. Die Verbindung der Akzentsetzung mit der Phonetik einer bestimmten Sprache, in der gesungen wird, verursacht, dass in einer Übersetzungssituation unterschiedliche Akzentuierung des Ausgangs- und des Zieltextes zu Melodiestörungen im Translat führen könnte.

Eine andere, für die Übersetzung wichtige Frage ist die Zuordnung des Liedes zu einem entsprechenden Texttyp. Katharina Reiß unterscheidet vier übersetzungsrelevante Texttypen: den inhaltsbetonten, formbetonten, appellativen und audiomedialen Text (vgl. K. Reiß 1993: 19). Die Dichtung wird von Reiß zu formbetonten Texten klassifiziert, wobei der Begriff 'Form' als Methode der Inhaltsvermittlung verstanden wird. Die Form ruft bei den Empfängern einen bestimmten ästhetischen Effekt hervor (vgl. K. Reiß 1969: 81f.). Sie bedeutet für Reiß u.a. Rhythmus, Reim, Metapher, Sprichwörter und Metrik (vgl. K. Reiß 1986: 39). Im Vergleich zu formbetonten Texten werden die audiomedialen durch zwei Merkmale bestimmt (vgl. K. Reiß 1986: 49-52): einerseits durch die Verwendung der technischen Anlagen für Tonaufnahme und -empfang (im Falle des Liedes und Liedtexte ist das u.a. Radio) und anderseits durch die Verwendung der außersprachlichen Ausdrucksformen (für die Lieder bedeutet das die musikalische Begleitung und Melodie). In Bezug auf Liedtexte kann man sagen, dass

\footnotetext{
${ }^{2}$ Wir sind der Meinung, dass es unmöglich ist, das Lied als Dichtung zu betrachten. Hypothetisch gesehen sind das für uns zwei unterschiedliche Formen der künstlerischen Tätigkeit, die durch andere Merkmale konstituiert werden.
} 
wir mit einem audiomedialen, formbetonten Texttyp zu tun haben. Da solche formalen Eigenschaften wie Strophenbau, Reime, Metrum oder Refrain zu den wichtigsten Merkmalen von Liedern gehören, wird von uns das Lied als eine Mischform des formbetonten und audiomedialen Textes angesehen.

\section{Liedtexte aus übersetzerischer Sicht am Beispiel von „Wilcza zamieć““}

Die Einbeziehung der Lieder in die Gruppe der audiomedialen, formbetonten Texte verursacht eine Reihe von Übersetzungsproblemen. Das Wichtigste ist hier die präzise Bestimmung des Übersetzungsskopos. Katharina Reiß und Hans Johann Vermeer betonen, dass „Dominante aller Translation der Zweck (Skopos)“ sei. Die Verschiedenheit der translatorischen Zielsetzungen bedeutet auch die Verschiedenheit der anwendbaren Übersetzungsstrategien und -techniken (vgl. K. Reiß / H. Vermeer 1984: 134). Im Falle der Lieder besteht der Übersetzungsskopos in der Beibehaltung von musikalischen Eigenschaften des Ausgangstextes. Die Verwendung der außersprachlichen Ausdrucksformen verursacht, dass der Liedtext sich in zwei gegenseitig beeinflussende Ebenen gliedern lässt, d.h in die Wortebene und in die Musikebene. Es ist unmöglich die genannten Ebenen zu trennen, weil die Veränderungen auf der Wortebene auch die Veränderungen auf der Musikebene implizieren. Darüber hinaus begleitet der wörtlichen Gestaltung des Ausgangstextes die musikalische Liedgestaltung. Aus der übersetzerischen Sicht kann man vermuten, dass sich nur die Wortebene übertragen lässt. Die Musikebene sollte unverändert bleiben und könnte höchstens nur bearbeitet werden ${ }^{3}$.

Der Übersetzer soll dementsprechend nach der rhythmischen Äquivalenz des Ausgangs- und des Zieltextes streben. Um das zu erzielen, lässt sich in Bezug auf das ausgangssprachliche Versmaß die durch Koller definierte transferierende Übersetzungsmethode anwenden. Sie besteht darin, dass man versucht, „AS-Textelemente, die spezifisch in der AS-Kultur verankert sind, als solche in ZS-Text zu vermitteln" (vgl. Koller 1998: 211). Im Weiteren soll auch versucht werden, relative semantische Treue in Bezug auf den Ausgangstext zu erzeugen. Der nächste Schritt im Übersetzungsprozess ist die Feststellung der Invarianz. Sie wird u.a. durch Krzysztof Lipiński als die Gruppe „der ausgangstextuellen Elemente, die im Vergleich zum Zieltext unverändert bleiben“, definiert ${ }^{4}$. Bei der Übersetzung der Lieder sind das vor allem rhytmische und reimische Textgestaltung (die die musikalische Ebene des Werkes beeinflussen).

Im empirischen Teil dieses Beitrags befassen wir uns mit der Übersetzung des Liedtextes aus dem derzeit sehr populären Computerspiel „Wiedźmin 3: Dziki Gon“, um zu veranschaulichen, auf welche Probleme der Übersetzer stoßen kann und worauf er aufpassen muss, um einen relativen Übersetzungserfolg zu erzielen. Dabei werden wir uns auf drei Übersetzungsebenen konzentrieren: auf die referentielle [REF], formale [FOR] und konnotative [KON] Ebene. Für die Beschreibung der Beispiele verwenden wir die einsprachigen Wörterbücher [SZYM] und [DUD]. Bei der Beschreibung

\footnotetext{
${ }^{3}$ Zur Differenzierung zwischen Übersetzung und Bearbeitung vgl. M. Schreiber (1993).

${ }^{4}$ grupa „[...] wszystkich tych parametrów thumaczonego tekstu, które przy porównaniu translatu z oryginałem pozostają niezmienne“" (übrs. E.L., vgl. K. Lipiński 2004: 86).
} 
der Übersetzungstechniken werden wir uns auf die Vorschläge von Krzysztof Lipiński ${ }^{5}$ beziehen und bei der Beschreibung des ausgangs- und zielsprachlichen Vermaßes berücksichtigen wir die Versmaßtypologie von Głowiński, Okopień-Sławińska und Sławiński ${ }^{6}$.

\section{Praktische Beispielsanalyse: die Übersetzung des Liedes „Wilcza zamieć“ ins Deutsche $^{7}$}

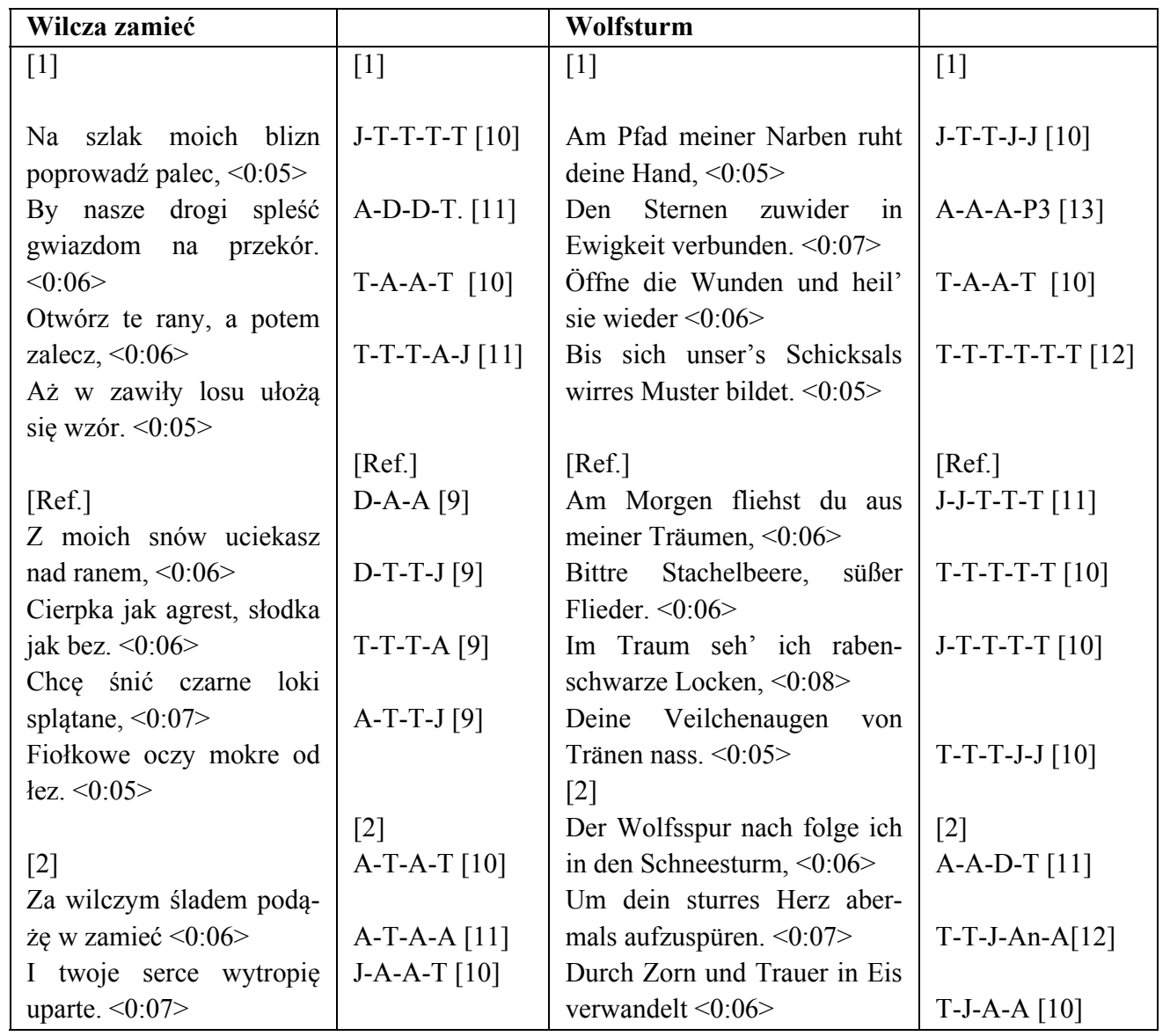

${ }^{5}$ Lipiński spricht in seinen Arbeiten über die sog. Substitutionen (darunter über Konkretisierung, Verallgemeinerung und Kompensation), Weglassungen, Ergänzungen und Inversionen (vgl. K. Lipiński 2004: 126 und 2002: 73).

${ }^{6}$ Die Autoren beschreiben Wortakzentmuster, indem sie solche Termini wie Trochäus, Jambus, Amphibrachus, Anapäst und Peon der Dritte (pl. Peon trzeci) verwenden (mehr dazu vgl. M. Głowiński/A. Okopień-Sławińska/J. Sławiński 1986: 169-173). Die genannten Begriffe werden auch in der Literaturwissenschaft zur Beschreibung des Dichtungsrhythmus angewendet.

${ }^{7}$ Aus ökonomischen Gründen werden bei der Beschreibung der formalen Liedebene folgende Abkürzungen verwendet: T - Trochäus, J - Jambus, D - Daktylus, A - Amphibrachus, An Anapäst, P3 - Peon 3. In $<>$ wird Artikulierungszeit der bestimmten Zeilen angegeben (gemessen von E.L.). 


\begin{tabular}{|c|c|c|c|}
\hline $\begin{array}{l}\text { Przez gniew i smutek } \\
\text { stwardniałe w kamień } \\
<0: 07> \\
\text { Rozpalę usta smagane } \\
\text { wiatrem. }<0: 05> \\
{[3]} \\
\text { Nie wiem, czy jesteś } \\
\text { moim przeznaczeniem, } \\
<0: 06> \\
\text { Czy przez ślepy traf mi- } \\
\text { tość nas związała? }<0: 07> \\
\text { Kiedy wyrzekłem moje } \\
\text { życzenie, }<0: 07> \\
\text { Czyś mnie wbrew sobie } \\
\text { wtedy pokochała? }<0: 06>\end{array}$ & $\begin{array}{l}{[3]} \\
\text { J-A-T-P3 [11] } \\
\text { T-T-A-T-T [11] } \\
\text { D-T-T-A [10] } \\
\text { D-T-T-P3 [11] }\end{array}$ & $\begin{array}{l}\text { Lass mich eine kalten Lippen } \\
\text { küssend schmelzen. }<0: 06> \\
{[3]} \\
\text { Ich frage mich, ob du mein } \\
\text { Schicksal bist, }<0: 06> \\
\text { Oder ob der Zufall uns zu- } \\
\text { sammenführte. }<0: 07> \\
\text { Als ich den letzten Wunsch } \\
\text { hervorbrachte, }<0: 06> \\
\text { Wurdest du gezwungen, mich } \\
\text { für immer zu lieben? }<0: 06>\end{array}$ & $\begin{array}{l}{[3]} \\
\text { A-T-T-D [10] } \\
\text { T-T-D-D-T [11] } \\
\text { J-J-J-T-T [10] } \\
\text { T-T-T-T-T-A [13] }\end{array}$ \\
\hline
\end{tabular}

\section{Zu Übersetzungstechniken auf der [FOR]-Ebene}

Auf der [FOR]-Ebene lässt sich feststellen, dass der Ausgangstext eine feste Reimstruktur besitzt, die aus Kreuzreimen besteht. Im Zieltext wird auf die gereimte Version verzichtet. Beim Vergleich des Ausgangstextes mit dem Zieltext ist die Verwendung unterschiedlichen Versmaßes zu sehen. Die Unterschiede sind auch in der Verslänge vorhanden. Die zielsprachlichen Zeilen sind häufig länger als die ausgangssprachlichen, was Probleme beim Singen verursachen kann, da Musik an die Deklamationszeit des Liedtextes angepasst wird. Zusammenfassend lässt sich feststellen, dass die musikalische Gestaltung des Ausgangstextes neutralisiert wurde. Trotz der Neutralisationen auf der [FOR]-Ebene wird die deutsche Übersetzung durch viele Empfänger ${ }^{8}$ als eine ästhetisch gelungene Wiedergabe angesehen. Man kann dementsprechend schlussfolgern, dass die metrische Gestaltung des Liedtextes die Melodik des ganzen Liedes nicht beeinflusst.

Die Artikulierungszeit der ausgangssprachlichen Zeilen ähnelt der Artikulierungszeit von zielsprachlichen Zeilen. Die Artikulation einzelner Zeilen vom Ausgangstext wird durch die polnische Sängerin verlängert, so dass der Endeffekt auf den Zuhörer gekünstelt wirken kann, weil das phonologische System im Polnischen keine Vokallänge berücksichtigt. Die Artikulationsverlängerung der polnischen Vokale durch die Sängerin hat wahrscheinlich dazu beigetragen, dass es fast keine Unterschiede in der Artikulationszeit der ausgangs- und zielsprachlichen Liedzeilen gibt, obwohl zieltextuelle Verse mehr Silben besitzen. Die Existenz der langen und der kurzen Vokale in der deutschen Sprache hat nämlich der Sängerin ermöglicht, den Liedtext besser an die melodischen Eigentümlichkeiten des Musikstückes anzupassen (was im Falle des Originalwerkes nicht zu hören ist), so dass der Zieltext dem deutschen Empfänger besser als Originalwerk erscheinen kann.

\footnotetext{
${ }^{8} \mathrm{http}$ //forum.worldofplayers.de/forum/threads/1436169-evtl-kleiner-Spoiler-Priscilla-s-SongSchönes-Ingame-Konzert (Zugriff am 02.05.2016)
} 


\section{Zu Übersetzungstechniken auf der [REF]-Ebene}

Auf der [REF]-Ebene bedient sich der Übersetzer außer wörtlicher Übertragung ${ }^{9}$ verschiedener Übersetzungstechniken, die unten beschrieben werden.

\section{Weglassung auf der [REF]-Ebene}

Weglassungen bedeuten laut Lipiński (2002: 73) den Verzicht auf die Einfügung eines Äquivalents in den Zieltext. Das passiert vorwiegend, um reimische oder rhythmische Struktur des Ausgangstextes in der Zielsprache beizubehalten.

Die zweite Zeile der ersten Strophe („by nasze drogi spleść gwiazdom na przekór“) wurde als „den Sternen zuwider in Ewigkeit verbunden“ übersetzt. Die Präpositionalphrase den Sternen zuwider fungiert hier als wörtliche Entsprechung der Übersetzungseinheit gwiazdom na przekór. Sowohl die ausgangsprachliche als auch die zielsprachliche Zeile beziehen sich auf den ersten Vers der ersten Strophe des Liedes und beschreiben die schicksalswidrige Liebesbeziehung. Der Übersetzer verzichtet zwar auf die Verwendung des wörtlichen Äquivalents polnischer Übersetzungseinheit by nasze drogi spleść (dt. um unsere Wege zu verbinden), aber er ergänzt zugleich den zielsprachlichen Text um neue Informationen, indem er ins Translat das Substantiv Ewigkeit einfügt.

Die zweite Zeile des Refrains („Cierpka jak agrest, słodka jak bez“) wurde mit Hilfe der Entsprechung bittre Stachelbeere, süßer Flieder übertragen. Die deutschen Substantivgruppen sind zwar als wörtliche Entsprechungen der polnischen Nominalphrasen zu interpretieren, aber der Übersetzer verzichtet auf die Übertragung des Konjuktions jak (dt. wie). Der Vezicht auf den Vergleich verursacht, dass die im Ausgangstext ausgedrückte Personifizierung gemildert wird, was zur Verallgemeinerung auf der $[R E F]-E b e n e ~ f u ̈ h r t{ }^{10}$. Um die o.g. Personifizierung im Translat beizubehalten, könnte man folgende Entsprechung vorschlagen: „die Bittere wie Stachelbeere, die Süße wie Flieder".

\section{Ergänzung auf der [REF]-Ebene}

Ergänzungen bedeuten immer zieltextuelle Einfügung neuer Elemente, die keinen Übersetzungseinheiten im Ausgangstext entsprechen (vgl. K. Lipiński 2002: 73).

Die in der zweiten Zeile der ersten Strophe präsente Ergänzung (vgl. Strophe [1], Zeile $<2>$ ) verbindet sich mit der o. g. Weglassung. Der Übersetzer fügt nämlich ins Translat das Substantiv Ewigkeit ein. Die Einfügung des neuen Elements lenkt die Interpretation des ganzen Liedes auf eine neue Bahn, indem es betont wird, dass es sich um ewige Liebesbeziehung handelt. Die [REF]-Ebene wird konkretisiert.

\footnotetext{
${ }^{9}$ Aus den ökonomischen Gründen verzichten wir auf die Besprechung der wörtlich übersetzten Beispiele.

${ }^{10}$ Der Empfänger des Zieltextes sollte die Phrase „bittere Stachelbeere, süßer Flieder“ mit Yennefer konnotieren, die in Andzej Sapkowskis Romanen die Geliebte des Hauptprotagonisten ist. Die übersetzerische Personifizierung ist deswegen nicht gelungen, weil das Substantiv Flieder einen männlichen Genus aufweist, der mit dem Sexus der personifizierten Person nicht übereinstimmt. Die Verwendung von Konjunktion wie ist deswegen unerlässlich.
} 
Die polnische Verbform zalecz (2. Pers. Sing. Imperativ, vgl. Strophe [1], Zeile $<3>$ ) wurde als heil' sie wieder übertragen. Die polnische Übersetzungseinheit wird in [SZYM] auf folgende Weise erläutert: „den Entwicklungsprozess einer Krankheit tilgen, eine Krankheit nicht zu Ende heilen" ${ }^{\text {"11 }}$. Sie wird auf das Substantiv rany bezogen, dessen wörtliche Entsprechung das Nomen Wunden ist. Da sich der Übersetzer entschieden hat, ins Translat ein Adverb einzufügen, das auf keine Übersetzungseinheit Bezug nimmt, bedeutet das deutsche Äquivalent im Kontext des Liedes noch einmal heilen.

Die polnische Substantivgruppe moje życzenie (vgl. Refrain, Zeile $<3>$ ) wurde als den letzten Wunsch übertragen. Der Übersetzer verwendet ein neues Lexem letzt, das die [REF]-Ebene des Zieltextes konkretisiert.

\section{Ergänzende Substitution auf der [REF]-Ebene}

Die Übersetzungseinheit stwardniate w kamień (vgl. Strophe [2], Zeile $<3>$ ) wird auf das Substantiv serce (dt. Herz, vgl. Strophe [2], Zeile <2>) bezogen und als ins Eis verwandelt übertragen. Die polnische Übersetzungseinheit bezieht sich auf den Phraseologismus ktoś ma serce z głazu, der u.a. bei [SZYM] als ,jmd. ist unzärtlich, rücksichtslos" beschrieben wird ${ }^{12}$. Der Übersetzer substituiert das polnische Substantiv kamień mit dem deutschen Nomen Eis (wörtliche Entsprechung im Polnischen: lód). Im Deutschen verzichtet man dadurch auf eine praseologische Bezugnahme. Darüber hinaus führt das auf der [REF]-Ebene des Ausgangs- und des Zieltextes zu Unterschieden in der Darstellungsweise.

\section{Verallgemeinerung auf der [REF]-Ebene}

Verallgemeinerungen sind laut Lipiński (2004: 126) ein Bestandteil der Substitution (d.h. des Ersatzes von Übersetzungseinheiten in der Zielsprache) und bestehen in der Verwendung eines Äquivalents mit der allgemeineren Bedeutung im Vergleich zum Ausgangstext.

Das Substantiv palec (vgl. Strophe [1], Zeile $<1>$ ) wird in [SZYM] als ,eines der fünf beweglichen Glieder einer Hand oder eines Füßes bei Menschen oder Tieren (Finger oder Zehe) “13 erläutert. Die wörtliche, deutsche Entsprechung im angegebenen Kontext des Liedes bedeutet Finger. Mit der Verwendung des Substantivs Hand hat der deutsche Übersetzer zur Verallgemeinerung des Zieltextes beigetragen.

Die Übersetzungseinheit nie wiem (vgl. Strophe [3], Zeile <1>) wurde als ich frage mich übertragen. Die polnische Negation besteht aus dem Negationswort nie (dt. nicht) und dem Verb wiedzieć (1. Per. Sing., dt. wissen). Der Übersetzer entscheidet sich allerdings für die Verwendung des Äquivalents ich frage mich (vgl. [DUD]: „sich etw.

${ }^{11}$ [SZYM]: „lecząc zatrzymać proces rozwoju choroby; chwilowo, niezupełnie wyleczyć (chorobę), powierzchownie zagoić (ranę)".

12 [SZYM]: „ktoś nie ulega wzruszeniom, jest nieczuły, niewrażliwy na coś, bezwzględny“. Das Substantiv głaz wird bei [SZYM] als „ein sehr großer Stein, Fels“ definiert (pl. „bardzo duży kamień, skała“).

${ }^{13}$ [SZYM]: ,jeden z pięciu członków stanowiących zakończenie ręki lub stopy człowieka; także zakończenie kończyn u zwierząt i ptaków; palec u ręki; palec u nogi”". 
überlegen, über etw. nachdenken, auf etw. neugierig, gespannt sein“), was der ganzen Äußerung einen indirekten Ton verleiht. Es wird nämlich implizit mitgeteilt, dass der Sender etwas nicht weiß.

\section{Konkretisierung auf der [REF]-Ebene}

Konkretisierungen sind wieder eine Ausdrucksform der Substitution; die Entsprechung weist einen ausführlichen Bedeutungsumfang im Vergleich zum Ausgangstext (vgl. K. Lipiński 2004: 126) auf.

Die verbale Phrase rozpale twe usta (vgl. Strophe [2], Zeile $<4>$ ) wurde kreativ als lass mich deine kalten Lippen schmelzen übertragen. Die Übersetzungsentscheidung ist die Folge der ergänzenden Substitution, die oben beschrieben worden ist. Der Übersetzer nimmt nämlich Bezug auf das ins Translat eingefügte Substantiv Eis (vgl. Strophe [2], Zeile $<3>$ ), indem er das Verb rozpale (dt. entflammen) mit Hilfe eines Antonyms schmelzen überträgt. Die [REF]-Ebene des Translats wird um eine neue Metapher konkretisiert.

Die Übersetzungseinheit czyś mnie wbrew sobie (vgl. Strophe [4], Zeile <4>) hat man als wurdest du gezwungen übertragen. Anstatt die wörtliche Entsprechung hast du mich wider deines Willens geliebt zu verwenden, entscheidet sich der Übersetzer für die Verwendung des unpersönlichen Äquivalents wurdest du gezwungen. Die übersetzerische Entscheidung hat einen Einfluss auf die Konkretisierung der [REF]-Ebene des Translats, weil sie suggeriert, dass fremde Personen die beschriebene Liebesbeziehung beeinflusst haben.

Die Substantivgruppe czarne loki (vgl. Refrain, Zeile $<3>$ ) wurde als rabenschwarze Locken übertragen. Der Übersetzer konkretisiert den Zieltext, indem er zum Adjektiv das Bestimmungswort raben- einfügt, das die Dunkelheit der Harre betont ${ }^{14}$.

\section{Zu Übersetzungstechniken auf der [KON]-Ebene}

Im Liedtext sind einige Anspielungen auf das Werk Andrzej Sapkowskis enthalten. Alle intertextuellen Elemente ${ }^{15}$ beziehen sich auf die Aussehensbeschreibung von Yennefer, die eine Geliebte des Protagonisten Geralt von Riva ist. Es entsteht dementsprechend die Frage, ob der zielsprachliche Empfänger imstande wird, die enthaltenen Anspielungen zu entschlüsseln. Das passiert jedoch unter zwei Voraussetzungen: der Empfänger soll die Werke Andrzej Sapkowskis kennen (die leserbedingte Voraussetzung) und der Übersetzer soll sich auf die dem zielsprachlichen Empfänger bekannten Übersetzungen beziehen (die übersetzerbedingte Voraussetzung).

Beispiel 1:

pl. Cierpka jak agrest, słodka jak bez - dt. Bittere Stachelbeere, süßer Flieder

\footnotetext{
${ }^{14}$ vgl. [DUD]: ,schwarz wie ein R. /die -n (ugs.: 1. sehr dunkel, tiefschwarz. 2. oft scherzh.; [meist von Kindern] sehr schmutzig).“

${ }^{15}$ Die beschriebenen intertextuellen Elemente betonen den intersemiotischen Charakter des Intertextualitätbegriffes, der besonders in der Computer- und Internet-Ära sichtbar wird. Mehr zu diesem Thema ist bei Sulikowski (2016) zu lesen.
} 
Die konnotativen Funktionen erfüllen im angeführten Beispiel zwei Substantive - agrest und bez. Sie werden als eine Anspielung auf das von Zauberin Yennefer beliebtes Pärfum interpretiert. Im ersten Band der Hexer-Saga ist Folgendes zu lesen:

Die Schulter der Zauberin war warm. Der schwarze Samt des Kleides verlangte geradezu nach einer Berührung. Der Geruch von Flieder und Stachelbeeren war angenehm betörend (A. Sapkowski 2016: 342) ${ }^{16}$

Die Substantive Flieder und Stachelbeere sind die wörtlichen Entsprechungen der Übersetzungseinheiten agrest und bez. Die Konnotation mit dem Yennefers Parfüm lässt sich auch im zielsprachlichen Lied entschlüsseln, weil in der Übersetzung des Romans von Andrzej Sapkowski auch die wörtlichen Äquivalente angewendet wurden.

Beispiel 2:

pl. Chcę śnić czarne loki splątane - dt. Im Traum seh' ich rabenschwarze Locken

Das nächste Beispiel ist die o. g. Konkretisierung. Sie bezieht sich auf die Haarfarbe von Yennefer. Dazu das entsprechende Textsegment:

Yennefer war sehr schön (...) Ihre rabenschwarzen Locken, die in Kaskaden auf die Schultern fielen, glänzten, warfen das Licht wie Pfauenfedern zurück, wanden sich und wogten bei jeder Bewegung. (A. Sapkowski 2016: 334) 17

Ähnlich wie beim letzten Beispiel ist die Konkretisierung eine direkte Anspielung auf das Werk Andrzej Sapkowskis, die sich wegen einer wörtlichen Übersetzung in der Zielkultur entschlüsseln lässt.

Beispiel 3:

pl. Fiołkowe oczy mokre od łez - dt. Veilchenaugen von Tränen nass

Das vorliegende Beispiel stellt die Anspielung auf die Augenfarbe Yennefers dar. Beim Vergleich des Andrzej Sapkowski Romans mit seiner Übersetzung erweisen sich einige Unterschiede:

Nein, darum beneidete sie Yennefer nicht, das wollte sie nicht gern haben und nicht einmal anschauen. Diese Augen, veilchenblau, tief wie unergründliche Seen (...)“ (A. Sapkowski 2016: 335$) 18$

\footnotetext{
${ }^{16}$ Vgl. polnische Textpassage: „Ramię czarodziejki było ciepłe. Czarny aksamit sukni aż prosił się o dotknięcie. Zapach bzu i agrestu rozkosznie oszałamiał” (A. Sapkowski 1999: 265).

${ }^{17}$ Vgl. polnische Textpassage: ,Yennefer była bardzo piękna (...) Jej kruczoczarne loki, kaskadą opadające na ramiona, lśniły, odbijały światło jak pawie pióra, wijąc się i falując przy każdym poruszeniu." (A. Sapkowski 1999: 259).

${ }^{18}$ Vgl. polnische Textpassage: „Nie, tego jednego nie zazdrościła Yennefer, tego jednego nie pragnęła mieć i nawet nie życzyła sobie oglądać. Tych oczu, fiołkowych, głębokich jak bezdenne jeziora (...)” (A. Sapkowski 1999: 259).
} 
Um die adjektivische Form der Augenbeschreibung von Yennfer beizubehalten, hat sich der Romanübersetzer Erik Simon für die Verwendung des Äquivalents veilchenblau entschieden. Das Lexem Veilchen fungiert dabei als eine wörtliche Entsprechung des polnischen Substantivs fiotek und besitzt laut [DUD] keine Adjektivform. Um daraus ein Adjektiv zu bilden, hat der Übersetzer ein zusätzliches Lexem blau ins Translat eingefügt, das die Augenfarbe der Zauberin konkretisiert. Die wörtliche Übersetzung der im ausgangssprachlichen Liedtext vorhandenen Substantivgruppe neutralisiert die [KON]-Ebene des Translats und kann beim zielsprachlichen Empfänger einen Verfremdungseffekt verursachen.

Beispiel 4:

pl. Kiedy wyrzekłem moje życzenie - dt. Als ich den letzten Wunsch hervorbrachte

Das letzte Beispiel ist die o. g. Ergänzung. Der Übersetzer entscheidet sich für die Verwendung des Lexems, das keiner Übersetzungseinheit im Ausgangstext entspricht. Die Substantivgruppe der letzte Wunsch (pl. ostatnie życzenie) ist eine Anspielung auf den Titel einer Erzählung, die das erste Treffen von Geralt und Yennefer schildert (vgl. A. Sapkowski 1998:220-276). Der deutsche Titel der genannten Erzählung ist eine wörtliche Übertragung (vgl. A. Sapkowski 2007:293-368). Die übersetzerische Entscheidung konkretisiert dementsprechend die [KON]-Ebene des Zieltextes.

\section{Schlussbemerkungen}

Im folgenden Beitrag wurde das Problem der Liedübersetzung am Beispiel des Liedtextes „Wilcza zamieć“ und ihrer deutschen Übersetzung angesprochen. Die Lieder wurden dabei als audiomediale, formbetonte Texte erfasst. Die untersuchte Übertragung wurde auf drei Ebenen beschrieben: auf der formalen [FOR], referentiellen [REF] und konnotativen [KON]. Die Untersuchung hat gezeigt, dass es am schwierigsten war, Äquivalenz an der [FOR]-Ebene zu gründen. Obwohl der Ausgangs- und Zieltext Unterschiede im Bereich des Rhythmus' und der Silbenlänge aufweisen sowie keine Reime besitzen, wird die deutsche Übertragung durch ihre Empfänger als eine künstlerisch gelungene Übersetzung beurteilt ${ }^{19}$. Daraus kann man schlussfolgern, dass die metrische Gestaltung des Liedtextes keinen Einfluss auf die melodische Gestaltung des ganzen Musikstückes hat. Die größte Rolle für die positive Beurteilung der [FOR]Ebene im Deutschen hat das Vorkommen von langen und kurzen Vokalen im Deutschen gespielt und die Tatsache, dass die Artikulation einiger Zeilen vom polnischen Liedtext verlängert wurde, was den Ausgangstext gekünstelt hat. Den anderen Grund dafür, dass phonologische Eigenschaften der deutschen Sprache die positive Beurteilung von [FOR] des Translats beeinflusst haben, liefert auch die wörtliche Übersetzung als die am meisten verwendete Übersetzungstechnik. In Bezug auf die [REF]-Ebene des Zieltextes hat der Übersetzer 13 aus 24 Zeilen des Ausgangstextes (54\%) wörtlich übertragen. In vier Zeilen (16\%) treten in der Zielsprache Konkretisierungen auf und zwei Zeilen (8\%) wurden zielsprachlich verallgemeinert. Darüber hinaus wurden drei

19 Vgl. u.a. http://forum.worldofplayers.de/forum/threads/1436169-evtl-kleiner-SpoilerPriscilla-s-Song-Schönes-Ingame-Konzert (Aufruf am 02.05.2016) 
Zeilen (12\%) ergänzt und im Falle von zwei Zeilen (8\%) hat der Übersetzer Weglassungen angewendet. Die Anwendung der wörtlichen Übersetzungstechnik beeinflusst die wörtlich-imitative Übersetzungsstrategie, was zur Neutralisation der formalen Translationsebene beiträgt. Die Folge dieser Übersetzungsstrategie ist die größere Treue auf der [REF]- und [KON]-Ebene des Translats.

\section{Bibliographie}

Benjamin, W. (1923), Die Aufgabe des Übersetzers. In: Gesammelte Schriften IV/1, Frankfurt/ M., 9-21.

Burdorf, D. (1997), Einführung in die Gedichtanalyse, Stuttgart, Weimar.

Deutsches Universalwörterbuch DUDEN (2007), Mannheim-Leipzig-Wien-Zürich. [DUD] Głowiński, M./ A. Okopień-Sławińska/J. Sławiński (1986), Zarys teorii literatury, Warszawa.

Jakobson, R. (1959), On linguistic aspects of translation. In: M. Baker/L. Venuti (Hrsg.) (2000), The Translation Studies Reader. London/ New York, 113-119.

Jakobson, R. (1960), Linguistik und Poetik. In: E. Holenstein/T. Schelbero (Hrsg.) (1979), Ausgewählte Aufsätze 1921-1971. Frankfurt/ M., 83-119.

Koller, W. (1998), Übersetzungen ins Deutsche und ihre Bedeutung für die Sprachgeschichte. In: W. Besch (1998), Sprachgeschichte: Ein Handbuch zur Geschichte der deutschen Sprache und ihrer Erforschung. Berlin/ New York, 210-228.

Lipiński, K. (2002), Po szynach ospale... O thumaczeniach Lokomotywy Juliana Tuwima na język niemiecki. In: J. Koźbiał (Hrsg.), Recepcja - transfer, przekład 1. Warszawa, 69-86.

Lipiński, K. (2004), Vademecum tlumacza. Kraków.

Reiß, K. (1981, 1969): Textbestimmung und Übersetzungsmethode. In: W. Wills (Hrsg.), Übersetzungswissenschaft. Darmstadt, 76-89.

Reiß, K./H. J. Vermeer (1984), Grundlegung einer allgemeinen Translationstheorie. Tübingen.

Reiß, K. (1986), Möglichkeiten und Grenzen der Übersetzungskritik: Kategorien und Kriterien für eine sachgerechte Beurteilung von Übersetzungen. München.

Reiß, K. (1993), Texttyp und Übersetzungsmethode. Der operative Text. Heidelberg.

Sapkowski, A. (1998), Ostatnie życzenie. Warszawa.

Sapkowski, A. (1999), Krew elfów, Warszawa.

Sapkowski, A. (2007), Der letzte Wunsch. München.

Sapkowski, A. (2008), Das Erbe der Elfen. München.

Schreiber, M. (1993), Übersetzung und Bearbeitung: Zur Differenzierung und Abgrenzung des Übersetzungsbegriffs. Tübingen.

Sulikowski, P. R. (2016), Der literarische Text und I-Faktoren in der Übersetzung. Anhand ausgewählter Werke Zbigniew Herberts im Deutschen und Polnischen. Frankfurt a. Main.

Szymczak, M. (1981), Stownik języka polskiego. Warszawa. [SZYM]

Wiedźmin 3: Dziki Gon, Hersteller: CD-Projekt RED, Polen 2015. (http://forum.worldofplayers.de/forum/threads/1436169-evtl-kleiner-SpoilerPriscilla-s-Song-Schönes-Ingame-Konzert; Zugriff: 02.05.2016) 